\title{
APRECIANDO SIMILARIDADES E VALORIZANDO DIFERENÇAS: Um Estudo sobre Atitudes de Gestores em Relação ao Diverso
}

\section{APPRECIATING SIMILARITIES AND VALUING DIFFERENCES: A Study on Managers' Attitudes toward the Diverse}

\author{
Darcy Mitiko Mori Hanashiro \\ Universidade Presbiteriana Mackenzie \\ darcyhanashiro@gmail.com
}

Marcelo Albuquerque Universidade Presbiteriana Mackenzie marcst_br@yahoo.com.br

Submissão: $31 / 07 / 2017$

Aprovação: 13/11/2017

\section{RESUMO}

Este artigo objetiva analisar as atitudes de gestores brasileiros em relação ao diverso (Universal-Diverse Orientation - UDO) e seus componentes atitudinais - diversidade de contato (DC), apreciação relativista (AR) e conforto com diferenças (CD) - e sua diferenciação por sexo, raça, idade e experiência no trabalho. A escala Miville-Guzman Universality-Diversity Scale-Short (M-GUDS-S), que mensura o constructo UDO, foi aplicada a uma amostra de 484 gestores de uma corporação multinacional do segmento de agronegócios. Foram obtidos valores satisfatórios de confiabilidade e validade convergente e discriminante da escala. De forma geral, os gestores apresentaram elevadas atitudes (UDO), revelando que são capazes de construir conexões, ser mais abertos e expressar mais sensibilidade com pessoas culturalmente diversas. Os testes estatísticos não foram significativos para sexo e raça. No entanto, houve uma correlação significativa e negativa entre idade e UDO global e DC, e entre experiência de trabalho e AR. Os resultados deste estudo têm implicações para a gestão da diversidade nas organizações, especificamente para subsidiar programas de treinamento, de expatriação e de carreira.

Palavras-chave: Atitudes em relação ao diverso. Diversidade. UDO. M-GUDS-S. 


\section{ABSTRACT}

This article aims to analyze the attitudes of Brazilian managers toward the diverse (Universal Diverse Orientation - UDO), their attitudinal components - diversity of contact (DC), relativistic appreciation, (RA) and comfort with differences (CD) - and their differentiation by sex, race, age and work experience. The Miville-Guzman Universality-Diversity Scale-Short (M-GUDS-S), which measures the UDO construct, was applied to a sample of 484 managers of an agribusiness multinational company. Satisfactory values of reliability and convergent and discriminant validation were obtained. In general, managers presented high scores of UDO, meaning that they are able to build connections, be more open and express more sensitivity toward culturally diverse people. Statistical tests were not significant for sex and race. However, there was a positive and significant correlation between age and global UDO and DC, and between work experience and RA. The results of this study have implications for managing diversity in organizations, especially to support training, expatriation and career programs.

Keywords: Attitudes toward the diverse. Diversity. UDO. M-GUDS-S.

\section{INTRODUÇÃO}

As organizações estão experienciando um aumento da diversidade na composição demográfica de seus empregados. De um lado, a globalização econômica e o desenvolvimento da tecnologia de informação e comunicação sem precedentes permitiram a ampliação das fronteiras de negócio e trabalhos com equipes multiculturais. De outro lado, forças internas adicionam novos desafios. A sociedade brasileira mostra-se mais heterogênea em termos de raça, idade, religião e orientação sexual. Essas mudanças trazem implicações diversas para a competitividade das organizações e, particularmente, para seus gestores, que necessitam estar mais bem preparados para atuar nessa nova realidade. Este fenômeno tem representado um dos maiores desafios para as lideranças, que passaram a ter que compreender a dinâmica da diversidade (MOR-BARAK, 2005), bem como seus efeitos e resultados para os negócios (FRANÇA; LOURENÇO, 2010). Como resultado, mais organizações têm adotado uma gestão da diversidade.

A efetividade das políticas de diversidade no ambiente de trabalho, contudo, depende das atitudes dos empregados em relação às pessoas provenientes de grupos de identidade distintos daqueles tradicionalmente majoritários nas organizações. Conhecer essas atitudes, portanto, é de vital importância para as corporações (PEREIRA; HANASHIRO, 2010), pois as atitudes precedem e predispõem os comportamentos, (FISHBEIN; AJZEN, 1975).

Dentre os estudos que envolvem a compreensão das atitudes em relação ao diverso, destaca-se o de Miville et al. (1999), no qual os autores realizaram várias pesquisas fundamentadas no constructo Orientação Universal ao Diverso, em inglês Universal-Diverse Orientation (UDO), que reflete uma atitude de conscientização e aceitação, tanto de similaridades como de diferenças existentes entre as pessoas. Similaridade remete ao universal, ou seja, àqueles aspectos do ser humano que são percebidos como sendo comuns; diferença refere-se aos aspectos que são únicos ou diversos entre pessoas, quando baseados em fatores culturais (por exemplo, raça, etnicidade, gênero, idade, e orientação sexual), assim como em fatores individuais (família de origem e funcionamento da personalidade). UDO é uma atitude social positiva com relação às similaridades e diferenças entre as pessoas; portanto, o constructo não remete a preconceito ou discriminação a membros de grupos minoritários ou diversos. O constructo UDO tem sua origem no campo da psicologia do 
aconselhamento, e é fundamentado principalmente na teoria de Vontress (1979, 1996). Miville et al. (1999) operacionalizaram o constructo mediante a escala Miville-Guzman Universality-Diversity Scale (M-GUDS) para mensurar as atitudes UDO.

O constructo UDO tem sido objeto de pesquisas, especialmente nos Estados Unidos, com estudantes universitários de graduação e pós-graduação. No Brasil, levantamento realizado nas bases de dados do Portal CAPES/MEC, no período de 01/01/90 a 23/07/2017, com as expressões Orientação Universal ao Diverso, UDO e M-GUDS-S, mostrou ausência de produção científica em periódicos envolvendo o constructo UDO, apesar de o estudo das atitudes em relação ao diverso ser um conhecimento fundamental, não apenas no ambiente universitário, mas também em organizações empresariais, para subsidiar a formulação de iniciativas voltadas à diversidade.

Diante desta lacuna no campo dos Estudos Organizacionais no Brasil e de sua importância, este artigo tem como objetivo analisar as atitudes UDO de gestores brasileiros e verificar se essas atitudes se diferenciam por sexo, raça, idade e experiência no trabalho.

A pesquisa foi realizada em uma amostra de gestores de uma corporação multinacional do segmento de agronegócios, atuantes em várias regiões do Brasil.

A pesquisa contribui para aprofundar a compreensão sobre as atitudes de gestores com relação ao diverso. Como contribuição prática, os resultados da pesquisa podem auxiliar as organizações na proposição de políticas de diversidade, tendo o UDO como instrumento de diagnóstico para avaliar possíveis resistências individuais às iniciativas de diversidade.

O artigo está organizado em quatro seções, além desta Introdução. Na primeira seção abordam-se o conceito de diversidade, a fundamentação do constructo UDO, o desenvolvimento da escala M-GUDS e a formulação das hipóteses. Na segunda seção são descritos os procedimentos metodológicos. Na sequência, são apresentados os resultados e sua discussão à luz da literatura, na terceira seção; finalmente, na quarta seção, apresentamos as conclusões, contribuições, limitações do estudo e propostas para futuras pesquisas.

\section{DIVERSIDADE}

Como resultado da diversificação do mercado de trabalho e globalização econômica, o local de trabalho, em geral, está mais heterogêneo, colocando o tema diversidade na agenda de interesse de gestores e pesquisadores de diferentes países. No Brasil, o cenário não é diferente e o mercado de trabalho tende a se tornar ainda mais diverso. As políticas públicas instituídas no Brasil nas últimas décadas, voltadas ao ensino superior, exercem impacto sobre o perfil demográfico do mercado de trabalho para universitários. O Fundo de Financiamento ao Estudante do Ensino Superior (FIES), o Programa Universidade para Todos (ProUni) e a Lei das Cotas para as instituições de ensino federal (Lei no 12.711, de 29 de agosto de 2012) são ações governamentais que ampliam a diversidade da força de trabalho no tocante à classe social, raça, diferenças regionais e culturais e experiência de vida. Pelo exposto, é incontestável supor que o ambiente de trabalho também esteja se tornando cada vez mais diverso.

O conceito de diversidade, no campo da gestão, começa a ganhar impulso, notadamente, com obras que se tornaram referências clássicas e seminais nos estudos de diversidade. Não raro, definições e dimensões de diversidade têm como referência recorrente Loden e Rosener (1991), Cox (1993), Thomas Jr. (1996), Nkomo e Cox (1999), e outros autores mais contemporâneos como Mannix e Neale (2005), Harrison e Klein (2007), Qin, Muenjoh e Chhetri (2014), entre outros.

As tentativas de conceituação da diversidade variam em amplitude, desde definições restritas, baseadas nas dimensões de raça, etnia e gênero, até definições extremamente amplas, 
que abrangem todas as diferenças entre as pessoas (HANASHIRO; CARVALHO, 2005). Outra forma de categorizar diversidade é em diferenças visíveis e menos visíveis (JACKSON; MAY; WHITNEY, 1995). As diferenças visíveis incluem raça, idade, gênero, deficiências físicas. As diferenças menos visíveis, também conhecidas como atributos subjacentes, incluem educação, habilidades, valores, atitudes, personalidade, tempo de casa, experiência profissional e orientação sexual. Apesar de discussões acerca do que constitui diversidade, Nkomo e Cox (1999) esclarecem que o termo se refere à diversidade de identidade.

As dimensões da diversidade são os traços que distinguem os sujeitos, e dizem respeito às diferentes identidades sociais dos indivíduos. Loden e Rosener (1991) adotam uma visão ampla, porém estabelecem distinções entre as dimensões primárias, que constituem diferenças humanas mais visíveis e praticamente imutáveis ao longo da vida, como idade, raça, sexo, habilidades físicas e orientação sexual; e diferenças secundárias, com traços menos observáveis e mais mutáveis, como formação educacional, experiências pessoais e profissionais, crenças religiosas, naturalidade geográfica, estado civil, e renda.

Nkomo e Cox (1999, p. 335) definem diversidade como "um misto de pessoas com identidades grupais diferentes dentro do mesmo sistema social". Assim, o conceito de identidade está na essência do entendimento da diversidade nas organizações, segundo os autores. Cox (1993) considera diferenças culturais aquelas originadas da identidade de nacionalidade, raça, etnia e gênero. A diversidade, em uma perspectiva mais ampla, também pode ser entendida como "qualquer atributo que outra pessoa pode usar para detectar diferenças individuais” (WILLIAMS; O'REILLY, 1998, p. 81), sem prévia categorização de dimensões, pois o que importa são as diferenças.

Outra forma de compreender a diversidade, além da perspectiva da identidade social, é defendida por Thomas Jr. (1996, p.7), para quem diversidade refere-se à "mistura coletiva de diferenças e similaridades ao longo de uma dada dimensão". Quando se lida com a diversidade, é importante focar a mistura coletiva e não apenas uma peça dessa mistura, ressalta o autor. As visões mais restritas da diversidade tendem a olhar apenas para uma ou algumas dimensões, como as diferenças. Na visão de Thomas Jr. (1996), contudo, entender a diversidade significa olhar o coletivo, que é formado por diferenças e também por similaridades.

Mais recentemente, Harrison e Klein (2007) apresentaram uma tipologia de diversidade, nomeadamente, separação, variedade e disparidade, com o propósito de comparar diferentes conceptualizações de diversidade. Qin, Muenjoh e Chhetri (2014) fizeram uma ampla revisão da literatura, examinando a variedade de conceituações de diversidade e propõem um modelo para unificar definições e compreensões fragmentadas de diversidade presentes na literatura.

A diversidade cultural no ambiente de trabalho tende a aumentar a criatividade das equipes, resultando em melhores decisões e vantagem competitiva frente a um mercado de consumidores que também é diverso (COX; BLAKE, 1991). De forma semelhante, Hanashiro e Carvalho (2005) sugeriram que grupos de trabalho heterogêneos tendem a apresentar vantagens na conquista de segmentos específicos de consumidores.

Ao tratar do tema diversidade, é importante ter sempre em mente a análise de como os grupos minoritários são afetados por comportamentos avessos à diversidade nos ambientes de trabalho (PEREIRA; HANASHIRO, 2011). Para as autoras, minorias são aqueles grupos que histórica e tradicionalmente foram sub-representados em poder, status, reconhecimento, recursos e prestígio. Dessa forma, os grupos de minoria são considerados diversos por serem os menos favorecidos, dominados economicamente e excluídos da pirâmide do poder político (FLEURY, 2000). 


\section{ORIENTAÇÃO UNIVERSAL AO DIVERSO (UDO)}

O constructo Orientação Universal ao Diverso, em inglês Universal-Diverse Orientation (UDO), teve origem no campo da Psicologia, em pesquisas sobre aconselhamento de estudantes de universidades nos Estados Unidos, e se refere à consciência e aceitação das semelhanças e diferenças entre as pessoas. Miville et al. (1999) desenvolveram o constructo UDO para analisar as condições de aceitação pelos indivíduos do diverso. Seus estudos partiram dos trabalhos sobre personalidade, de Jung (2000), mais especificamente sobre os arquétipos universais que os indivíduos herdam e que os conectam. Então, eles prosseguiram com os processos de Yalom (1985, apud Miville et al., 1999), pelos quais membros de grupos reconhecem qualidades universais que permitem compartilhar experiências. E, por fim, utilizaram os estudos de Vontress (1996), segundo os quais um aconselhamento psicológico eficaz requer que o conselheiro tenha consciência das semelhanças e diferenças entre as pessoas, como produtos de várias culturas - universal, ecológica, nacional, regional, e racioétnica - que interagem e as tornam, simultaneamente, semelhantes e diferentes em muitos aspectos.

A similaridade decorreria da cultura universal ou da condição biológica comum a todos os seres humanos, pois, independente das condições em que as pessoas vivem, os processos biológicos da vida conectam as pessoas enquanto seres humanos, o que permite a construção de alianças baseadas nas semelhanças entre elas (VONTRESS, 1996, p. 164). A diferença, por sua vez, decorreria das outras categorias de cultura: nacional, regional, ecológica, religiosa e racioétnica, que se referem às experiências vivenciadas por grupos diversos, e que também influenciam sua composição psicológica e sua capacidade de interação com os outros (VONTRESS, 1996).

O constructo UDO refere-se a uma atitude social positiva em relação às outras pessoas (MIVILLE et al., 1999), na qual um indivíduo com uma atitude positiva em relação à diversidade seria mais inclinado a aceitar aqueles que lhe são diferentes, ao passo que indivíduos com uma atitude negativa à diversidade seriam menos propensos a aceitar os que lhe são distintos (HOMAN et al., 2007). O termo universal do UDO se refere ao reconhecimento das semelhanças que conectam todos os seres humanos (cultura universal), enquanto o termo diverso trata dos aspectos exclusivos das pessoas, ou fatores individuais que impactam a capacidade dos indivíduos de interagir eficazmente (cultura diversificada).

O constructo UDO caracteriza-se pela inter-relação dos componentes cognitivo, comportamental e afetivo das atitudes. Ou seja, uma pessoa com atitudes favoráveis à diversidade universal que une os seres humanos (semelhanças e diferenças) tende a buscar uma diversidade de experiências com os outros (componente comportamental), porque valoriza tanto as semelhanças quanto as diferenças entre ela e os outros (componente cognitivo), em um contexto em que as experiências compartilhadas levam a um senso de conexão com os outros (componente afetivo ou emocional) (MIVILLE et al., 1999). Por atitude entende-se "uma predisposição para responder de forma favorável ou desfavorável a um objeto, pessoa, instituição ou acontecimento" (FISHBEIN; AJZEN, 1975, p.6).

Miville et al. (1999) desenvolveram uma escala para medir o constructo UDO: a Miville-Guzman Universality-Diversity Scale (M-GUDS). Os autores constataram que as escalas existentes para atitudes em relação à diversidade eram baseadas somente na medição de atitudes negativas, como discriminação e preconceito, em vez da mensuração de atitudes sociais positivas, como a abertura ao diverso e a aceitação dos outros.

A M-GUDS foi criada com 45 itens, composta por três subescalas: a) Apreciação relativista, de si próprio e dos outros, envolvendo reconhecimento e aceitação de similaridades e diferenças entre pessoas; b) Diversidade de contato, incluindo tanto comportamentos prévios como intencionais, relevantes para contatos com pessoas de 
diferentes experiências (background) demográficas (exemplo: raça, gênero e religião); e c) Senso de conexão com a sociedade mais ampla e a humanidade como um todo, envolvendo ligações emocionais que uma sente em relação a outras pessoas. Essas subescalas foram criadas para assegurar adequada representação de componentes inter-relacionados cognitivos, comportamentais e afetivos (MIVILLE et al., 1999). Os itens da M-GUDS são mensurados em uma escala no formato Likert de 6 pontos, variando de 1 (discordo fortemente) a 6 (concordo fortemente). Alguns itens foram expressos positivamente e alguns negativamente, para contrabalançar potenciais respostas enviesadas.

Miville et al. (1999) desenvolveram quatro pesquisas, todas realizadas com amostras heterogêneas de estudantes universitários dos Estados Unidos, com o objetivo de criar um instrumento para medir o constructo UDO e estabelecer evidências de confiabilidade e validade da M-GUDS. Os resultados desses quatro estudos indicaram que a M-GUDS tinha alto nível de confiabilidade (coeficiente alfa=0,94). Os escores de validade convergente e discriminante indicam que a M-GUDS é significativamente e positivamente relacionada a medidas de identidade racial, feminismo e androginia, e negativamente relacionada a homofobia e dogmatismo, conforme suposições teóricas. A validade discriminante teve resultados mistos em relação à desejabilidade social, evidenciando a necessidade de mais estudos. Miville et al. (1999) encontraram suporte para uma estrutura unidimensional da MGUDS, indicando que o escore total da escala, mais do que os escores das subescalas, poderia ser usado para refletir a natureza aparentemente unidimensional do instrumento. Assim, concluíram que a UDO é mais bem conceptualizada como um constructo unidimensional com componentes comportamental, cognitivo e afetivo, em vez de um constructo multidimensional com três distintos domínios inter-relacionados.

Fuertes et al. (2000) realizaram três estudos com amostras de estudantes de graduação e pós-graduação de universidades dos Estados Unidos, com o objetivo de examinar a estrutura fatorial da M-GUDS. O resultado foi uma proposta de versão reduzida, a Miville-Guzman Universality-Diversity Scale-Short (M-GUDS-S). Para os autores, esses estudos contribuíram para o refinamento na conceptualização e mensuração da UDO. A análise fatorial confirmatória sugere que a M-GUDS-S mede a UDO como um constructo multidimensional com três domínios distintos, mas modestamente inter-relacionados: comportamental, emocional e cognitivo. As três escalas da M-GUDS-S aparecem conceitualmente similares ao proposto por Miville et al. (1999). A despeito dessas similaridades, os três estudos de Fuertes et al. (2000) ajudaram a redefinir o componente afetivo associado à UDO. Os achados das pesquisas sugerem que essa subescala afetiva compreende itens que podem designar duas dimensões emocionais da UDO distintas, mas relacionadas: um senso de conexão com outros que lhe são diferentes, e ambivalência e desconforto potencial em relação a esse contato. Assim, para Fuertes et al. (2000) os resultados da pesquisa sugerem que o componente afetivo é mais complexo do que apresentado por Miville et al. (1999). Para refletir essa diferença, este fator foi denominado Conforto com Diferenças. A M-GUDS-S compreende 15 itens, cinco para cada dimensão.

A M-GUDS-S apresenta três vantagens para os pesquisadores em relação à escala MGUDS. Primeiro, a M-GUDS-S é menor e pode ser aplicada mais rapidamente que a escala original. Segundo, a estrutura fatorial da M-GUDS-S e a relação entre suas subescalas é mais claramente delineada. Terceiro, o uso da M-GUDS-S permite, para uma análise da UDO, usar os escores das subescalas, dado que eles medem aspectos distintos da UDO e predizem, diferentemente, atitudes e comportamentos em relação ao diverso (FUERTES et al., 2000).

Neste artigo foi utilizada a escala M-GUDS-S (FUERTES et al., 2000), pelas justificativas apresentadas.

A literatura aponta um crescimento das pesquisas que utilizam o constructo UDO em áreas não somente da Psicologia voltada ao aconselhamento. Verifica-se um gradual aumento 
de pesquisas científicas com foco em explorar, sobretudo, as variáveis preditoras da atitude UDO. Observa-se, contudo, que o sujeito de pesquisa continua sendo estudante de graduação ou pós-graduação. A título de apresentar a evolução dos estudos concernentes ao constructo, menciona-se um corpo de pesquisas que busca verificar algumas variáveis que influenciam as atitudes UDO, tais como: gênero, raça, personalidade e exposição à diversidade (STRAUSS; CONNERLEY, 2003); autoeficácia generalizada, otimismo e pensamento positivo, autoestima pessoal e coletiva e conexão social (MIVILLE et al., 2004); valores pessoais (SAWYERR; STRAUSS; YAN, 2005); traços de personalidade (ROBERTS; LAUX; BURCK, 2009); raça/etnia e gênero (SINGLEY; SEDLACEK, 2009); variáveis demográficas de estudantes universitários, características dos campi pesquisados, envolvimento em experiência prévia em diversidade e clima multicultural dos campi (TOSCANO, 2012); variáveis demográficas, diferenças geográficas, afiliação políticas, imersão em ambientes diversos por meio de oportunidades de viagem fora da cidade e do país, e número de cursos sobre diversidade (KOHLI et al., 2016).

\section{FORMULAÇÃO DAS HIPÓTESES}

O constructo UDO foi desenvolvido no campo da psicologia de aconselhamento, a fim de investigar as atitudes de conselheiros de alunos nas universidades multiculturais dos Estados Unidos. Desde sua criação, a literatura aponta estudos que buscaram entender a relação entre personalidade, estrutura de valores individuais, religiosidade e efeitos de variáveis demográficas, como variáveis independentes ou moderadoras, e a UDO como variável dependente, todos aplicados em estudantes universitários. Idade, raça e sexo são considerados dimensões primárias da diversidade, e determinam aspectos fundamentais da identidade social dos indivíduos, influenciando sua visão de mundo. Nesse sentido, é importante conhecer em que medida essas variáveis demográficas estariam associadas às atitudes dos indivíduos em relação ao diverso.

Alguns resultados de pesquisas internacionais mostram que as atitudes em relação ao diverso ou à diversidade são sensíveis em relação a sexo, raça e idade. As mulheres seriam mais favoráveis a ações voltadas à diversidade do que os homens, por terem um senso de auto interesse cooperativo em relação às iniciativas de diversidade (KLUEGEL; SMITH, 1983). As mulheres também tendem a valorizar mais os esforços dos colegas de trabalho em promover a diversidade do que os homens brancos (KOSSEK; ZONIA,1993). Nessa mesma linha, os achados de Mor-Barak (2000) apontam que não somente as mulheres, mas as minorias, tendem a ter uma atitude mais positiva em relação às políticas de ação afirmativa e diversidade que os homens. Em dois estudos realizados por Miville et al. (1999) com estudantes de universidades dos Estados Unidos, ficou evidenciado que as mulheres tiveram escores da escala M-GUDS levemente mais elevados que os escores dos homens. Resultados similares foram obtidos por Strauss e Connerley (2003) e Sawyerr, Strauss e Yan (2005), em amostras de estudantes universitários de diferentes regiões dos Estados Unidos; e também Strauss, Sawyerr e Oke (2008), em uma amostra de respondentes britânicos. Os resultados evidenciaram uma correlação positiva significativa (para mulheres) com UDO global e as subescalas Apreciação Relativista e Diversidade de Contato (STRAUSS; CONNERLEY, 2003; SAWYERR; STRAUSS; YAN, 2005) e Conforto com Diferenças (STRAUSS; SAWYERR; OKE, 2008).

Com base nessas evidências empíricas e nos argumentos teóricos anteriormente apresentados, foi proposta a seguinte hipótese:

Hipótese 1. As gestoras mulheres terão escores da M-GUDS-S (mensuração de atitudes de UDO) mais elevados que os escores dos gestores homens. 
Em relação à raça, os estudos têm mostrado que homens brancos têm atitudes menos favoráveis aos diversos e às iniciativas de diversidade. Fuertes et al. (2000) realizaram estudos sobre a UDO e constataram que os indivíduos asiático-americanos tinham pontuação UDO mais alta que homens brancos quanto à diversidade de contato, da mesma forma que Strauss e Connerley (2003) identificaram que os indivíduos não brancos demonstravam atitudes mais favoráveis ao diverso do que indivíduos brancos, principalmente no tocante à busca de contato com pessoas diferentes (dimensão diversidade de contato da UDO). Strauss, Sawyerr e Oke (2008) identificaram que os indivíduos não brancos demonstravam atitudes mais favoráveis à diversidade do que os brancos para a UDO em geral, e não em uma das dimensões do constructo. Linnehan et al. (2003) também realizaram estudos com grupos asiático-americanos e concluíram que sua forte identidade racial fazia com que eles fossem mais propensos que os estadunidenses brancos a iniciativas organizacionais mais pluralistas. Isto porque os grupos raciais que foram historicamente excluídos tendem a apoiar ações que signifiquem respeito à sua identidade racial, concluem os autores. Com base nessas constatações empíricas, foram formuladas duas hipóteses:

Hipótese 2a. Os gestores do sexo masculino não brancos terão escores da M-GUDS-S (medida de atitudes de UDO) mais elevados que os escores dos gestores brancos.

Hipótese 2b. As gestoras mulheres não brancas terão escores da M-GUDS-S (medida de atitudes de UDO) mais elevados que os escores das gestoras brancas.

Pesquisas sugerem que a idade pode fazer com que os indivíduos alterem suas atitudes frente à diversidade (LODEN; ROSENER, 1991). Strauss e Connerley (2003) constataram a existência de correlação positiva entre idade e atitudes UDO na dimensão UDO Conforto com Diferenças. Em pesquisas sobre valores, Schwartz (1992) constatou que pessoas mais jovens atribuíam maior importância a valores de abertura à mudança e menor ao conservadorismo do que pessoas mais velhas. Com base nessas evidências a seguinte hipótese foi formulada:

Hipótese 3. Os gestores mais velhos terão escores da M-GUDS-S (medida de atitudes de UDO) mais elevados que os escores dos gestores mais novos.

Forteza e Prieto (1994) defendem que a experiência dos indivíduos tende a influenciar suas reações cognitivas e afetivas no ambiente de trabalho, podendo assim influenciar as atitudes UDO dos gestores. Apesar de não tratar especificamente da variável idade, mas sim da experiência como gestor, é possível inferir que a variável Experiência no Trabalho guarda similaridade com a idade, uma vez que o ganho de experiência depende do passar do tempo e, em decorrência, do aumento da idade. Com base nessa inferência, foi elaborada a seguinte hipótese:

Hipótese 4. Os gestores com mais experiência terão escores da M-GUDS-S (medida de atitudes de UDO) mais elevados que os escores dos gestores com menos experiência.

\section{PROCEDIMENTOS METODOLÓGICOS}

A escala M-GUDS-S foi desenvolvida em Inglês e traduzida para o Português, de acordo com o processo de tradução reversa definido por Brislin (1970). Uma vez atingida a equivalência conceitual dos itens nos dois idiomas, foi realizada avaliação semântica e adaptação cultural da escala, de modo a garantir que os termos empregados no questionário estivessem coerentes com o sentido das experiências vivenciadas pelos indivíduos da amostra da pesquisa (GUILLEMIN; BOMBARDIER; BEATON, 1993). Na validação semântica e cultural foi constatado que dois itens da subescala Diversidade de Contato (DC) não estavam adequados ao contexto cultural brasileiro; assim, esses itens foram substituídos por outros dois itens da M-GUDS (45 itens) da mesma subescala (DC). Na sequência, foi realizado um pré-teste, para avaliar o nível de compreensão da escala, com um comitê revisor composto por 
20 juízes, sendo 10 homens e 10 mulheres, com idades entre 21 e 70 anos e a seguinte formação: quatro doutores, cinco mestres, sete especialistas, dois graduados e dois com graduação incompleta. Foi solicitado que os juízes lessem todos os itens e atribuíssem escores entre 1 (nada claro) e 10 (totalmente claro) para cada um. Os itens com escores inferiores a $70 \%$ foram revisados.

A organização onde foi realizada a pesquisa é uma corporação multinacional, do setor de agronegócios e alimentos, com 9.000 empregados, estabelecida no Brasil desde 1965 e presente em todas as regiões do país. A população-alvo do estudo foi composta por gestores nos cargos de Supervisão, Gerência e Direção, de todas as regiões do Brasil. Foram convidados a participar da pesquisa todos os gestores da empresa, ou seja, 1.425 pessoas. Foram respondidos 506 questionários, correspondendo a uma taxa de 35\% de retorno. O número de respostas atendeu plenamente aos critérios mínimos recomendados na literatura para garantir um nível adequado de poder estatístico da pesquisa (HAIR et al., 2005).

A coleta dos dados foi realizada por meio de um serviço de pesquisa da Internet denominado SurveyMonkey. Os gestores da empresa foram comunicados sobre a pesquisa por e-mail, enviado pela Diretoria de $\mathrm{RH}$, no qual foram enfatizados a importância do assunto, sua natureza científica, o caráter voluntário da participação e a total confidencialidade das respostas.

$\mathrm{O}$ apoio da Diretoria de RH suscitou preocupação em relação à integridade das respostas dos gestores, devido a uma possível condição de desejabilidade social. Esta é uma situação em que os participantes podem se sentir propensos a responder às questões de modo a negar sua associação pessoal com opiniões ou comportamentos socialmente reprovados (GOUVEIA et al., 2009). Para evitar essa influência foram adotados os seguintes remédios contra vieses em pesquisas comportamentais: 1) Foi enfatizada a garantia do anonimato aos participantes e reforçada a total liberdade para que interrompessem sua participação a qualquer tempo (PODSAKOFF; MACKENZIE; PODSAKOFF, 2003); 2) Foi informada a inexistência de respostas certas ou erradas, de modo que os participantes pudessem responder às perguntas da forma mais honesta e livre possível (PODSAKOFF; MACKENZIE; PODSAKOFF, 2003); 3) O questionário foi disponibilizado por meio de um serviço de pesquisas independente e externo à empresa (SurveyMonkey); e 4) Para identificar eventuais vieses nas respostas dos participantes, foram incluídos cinco itens indicativos de desejabilidade social extraídos da escala de Gouveia et al. (2009), com base nos valores de cargas fatoriais mais elevados e adequação de conteúdo.

\section{RESULTADOS}

Antes de realizar o tratamento estatístico, procedeu-se à limpeza do banco de dados. O tratamento de respostas em branco ou repetidas foi realizado conforme orientação da European Social Survey Education Net (EUROPEAN SOCIAL SURVEY EDUCATION NET, 2012). Os dados faltantes foram substituídos pela média das respostas do item (HAIR et al., 2005). Após esse procedimento, do total de 506 questionários respondidos restaram 484. Nas escalas M-GUDS-S e de desejabilidade social, itens reversos tiveram as respostas invertidas.

Uma análise de confiabilidade, validade convergente e discriminante da escala MGUDS-S foi realizada pelo modelo de mensuração do software SmartPLS 2.0.M3. Para a avaliação desses valores de qualidade foram seguidas as orientações apresentadas por Ringle, da Silva e Bido (2014).

1) Validade convergente: a) análise da Variância Média Extraída (AVE), que deve ser > $0,50 ; b)$ análise das cargas fatoriais que devem ser significantes (valores-t $>1,96$ ); c) análise das cargas fatoriais, que devem ser $>0,7$. 
2) Validade discriminante: a) no nível dos itens: observação das cargas cruzadas, em que as cargas fatoriais devem ser mais altas nas respectivas VL (subescalas da UDO) do que em outras VL; b) no nível dos constructos: observação da raiz quadrada da AVE que deve ser maior que a correlação entre as variáveis latentes (subescalas da UDO).

3) Confiabilidade: a) Análise da consistência interna (alfa de Cronbach), que deve ser > 0,7; b) Análise da confiabilidade composta, que deve ser $>0,7$.

Alguns comentários são necessários para explicar a decisão de manter todas as variáveis da escala M-GUDS-S, diante dos resultados não satisfatórios obtidos. Embora a validade convergente observada pela variância média extraída (AVE) tenha ficado inferior a 0,5 nas três subescalas, optou-se por manter todas as variáveis em vez de eliminar aquelas com cargas fatoriais (correlações) de menor valor para elevar a AVE. Conforme Pedhazur (1997), quando os valores não estão muito distantes do recomendado, deve-se tentar manter o maior número possível de variáveis da estrutura, em vez de comprometer a validade de conteúdo do constructo.

A validade convergente, pelas cargas fatoriais da escala M-GUDS-S, apresentou nove variáveis com cargas menores que 0,7 (menor valor=0,4726). Hair et al. (2014) defendem que, em pesquisas sociais, algumas cargas fatoriais podem ficar abaixo de 0,7. Assim, todas as variáveis foram mantidas de modo a não desconfigurar a validade de conteúdo da escala (PEDHAZUR, 1997). Da mesma forma, Chin e Newsted (1999) sugerem que um modelo que tenha apresentado uma carga fatorial baixa em uma amostra possa apresentar resultado diferente em outra, quando as amostras utilizadas forem significativamente diferentes, o que ocorreu com as amostras utilizadas no Brasil e nos Estados Unidos. A confiabilidade medida pelo alfa de Cronbach foi menor que 0,7 em AR e CD. Conforme Ringle, da Silva e Bido (2014), valores do alfa de Cronbach acima de 0,60 e 0,70 são considerados adequados em pesquisas exploratórias. Em suma, todos os itens da M-GUDS-S foram mantidos; entretanto, futuros estudos devem observar cuidadosamente a validade convergente, pois se as cargas fatoriais e valores da AVE se mantiverem baixos isto pode levar à retirada de alguns itens ou à sua revisão.

Os valores de referência e os resultados obtidos da análise são apresentados nas Tabelas 1 e 2 .

Tabela 1 - Confiabilidade e validade convergente e discriminante (no nível dos itens)

\begin{tabular}{|c|c|c|c|c|}
\hline & Valores referenciais & $\begin{array}{c}\text { Apreciação } \\
\text { relativista (AR) }\end{array}$ & $\begin{array}{c}\text { Conforto com } \\
\text { diferenças (CD) }\end{array}$ & $\begin{array}{l}\text { Diversidade de } \\
\text { contato (DC) }\end{array}$ \\
\hline \multirow{3}{*}{$\begin{array}{c}\text { Validade } \\
\text { convergente }\end{array}$} & AVE & 0,41 & 0,42 & 0,48 \\
\hline & Valor-t >1,96 & $\mathrm{t}>1,96$ & $\mathrm{t}>1,96$ & $\mathrm{t}>1,96$ \\
\hline & Carga fatorial $>0,7$ & 4 itens $<0,7$ & 3 itens $<0,7$ & 2 itens $<0,7$ \\
\hline \multirow{2}{*}{ Confiabilidade } & Alfa de Cronbach & 0,6358 & 0,6687 & 0,7289 \\
\hline & $\begin{array}{c}\text { Confiabilidade } \\
\text { composta }\end{array}$ & 0,7714 & 0,78960 & 0,8203 \\
\hline $\begin{array}{c}\text { Validade } \\
\text { discriminante }\end{array}$ & No nível dos itens & $\begin{array}{c}\text { Cargas fatoriais > } \\
\text { em AR }\end{array}$ & $\begin{array}{c}\text { Cargas fatoriais }>\mathrm{em} \\
\mathrm{CD}\end{array}$ & $\begin{array}{c}\text { Cargas fatoriais > } \\
\text { em DC }\end{array}$ \\
\hline
\end{tabular}

Fonte: Elaborado pelos autores. 
Tabela 2 - Validade discriminante no nível do constructo

\begin{tabular}{|c|c|c|c|c|}
\hline & & $\begin{array}{c}\text { Apreciação } \\
\text { relativista (AR) }\end{array}$ & $\begin{array}{c}\text { Conforto com } \\
\text { diferenças (CD) }\end{array}$ & $\begin{array}{l}\text { Diversidade de } \\
\text { contato (DC) }\end{array}$ \\
\hline \multirow{3}{*}{$\begin{array}{c}\text { Validade } \\
\text { discriminante no } \\
\text { nível dos } \\
\text { constructos }\end{array}$} & $\begin{array}{c}\text { Apreciação } \\
\text { relativista (AR) }\end{array}$ & 0,636 & & \\
\hline & $\begin{array}{l}\text { Conforto com } \\
\text { diferenças (CD) }\end{array}$ & 0,059 & 0,663 & \\
\hline & $\begin{array}{l}\text { Diversidade de } \\
\text { contato (DC) }\end{array}$ & 0,522 & 0,259 & 0,691 \\
\hline
\end{tabular}

Fonte: Elaborado pelos autores.

Nota: Os valores em negrito (na diagonal) são a raiz quadrada da AVE. Os demais valores são correlações entre as variáveis latentes (subescalas).

Uma preocupação com relação às respostas aos itens da M-GUDS é que os respondentes podem se comportar de forma positiva, com uma imagem mais tolerante em relação a si mesmos. Miville et al. (1999) incluíram em dois estudos a escala de desejabilidade social de Marlowe-Crowne - Social Desirability Scale. No primeiro não foi encontrada correlação entre a M-GUDS e desejabilidade social (DS). No segundo estudo, ao contrário do previsto, a M-GUDS correlacionou positivamente com a DS. Por esta razão, nesta pesquisa foram introduzidos cinco itens, como explicado anteriormente. A análise do efeito da DS foi realizada pela comparação entre a correlação bivariada das subescalas (DC, $\mathrm{AR}$ e $\mathrm{CD}$ ) e as correlações parciais controladas pela variável DS. O resultado indicou não haver efeito da DS, ou seja, os respondentes não responderam a M-GUDS-S de forma socialmente desejada.

A seguir são apresentados a descrição da amostra e os resultados das hipóteses definidas no estudo.

A amostra é composta por gestores do sexo masculino (71,9\%), brancos $(77,7 \%)$, com idades entre 30 e 49 anos $(73,8 \%)$, com tempo de trabalho como gestor variando entre 0 e 9 anos $(61,6 \%)$, com escolaridade de níveis Superior e Especialização $(79,1 \%)$, de religião católica $(63,2 \%)$, em sua maioria casados $(74,4 \%)$ e heterossexuais $(96,5 \%)$. Este perfil evidencia que, apesar de a empresa já ter realizado campanhas em prol da diversidade no passado, as características dos gestores que responderam à pesquisa ainda são consideravelmente homogêneas.

A M-GUDS-S avalia atitudes de consciência e de aceitação das similaridades e diferenças entre pessoas (MIVILLE et al., 1999). Um aspecto importante é poder realizar uma análise global e saber quais são as atitudes dos respondentes, se seus escores tendem para uma atitude UDO mais ou menos elevada, indicando serem mais favoráveis ou não ao diverso. As características sociodemográficas apontaram que os gestores respondentes da pesquisa configuram um grupo de maioria (mainstream) homogêneo nos quesitos sexo, idade, escolaridade e religião. As respostas se concentraram predominantemente nas categorias concordo e concordo fortemente, com um coeficiente de variação considerado baixo. Isso significa que os gestores têm uma atitude favorável em relação ao diverso, e essas respostas apresentam baixa dispersão e homogeneidade, portanto, sem grandes variações em torno da média, conforme mostrado na Tabela 3. 
Tabela 3 - Medidas de posição e de dispersão

\begin{tabular}{lcccc}
\hline Medidas & $\begin{array}{c}\text { Diversidade } \\
\text { de Contato }\end{array}$ & $\begin{array}{c}\text { Apreciação } \\
\text { Relativista }\end{array}$ & $\begin{array}{c}\text { Conforto } \\
\text { com } \\
\text { diferenças }\end{array}$ & UDO global \\
\hline Média & 5,13 & 4,85 & 5,28 & 5,09 \\
Moda & 5,60 & 5,00 & 5,80 & 5,07 \\
Desvio-padrão & 0,61 & 0,66 & 0,60 & 0,44 \\
Coeficiente de variação & $11,9 \%$ & $13,5 \%$ & $11,3 \%$ & $8,6 \%$ \\
\hline
\end{tabular}

Fonte: Elaborado pelos autores.

\subsection{Análise das hipóteses}

Uma análise da normalidade das variáveis foi necessária para verificar se a distribuição de probabilidade subjacente aos dados relativos às subescalas de UDO e UDO global pode ser aproximada pela distribuição normal. Para esta finalidade foi aplicado o teste de normalidade de Kolmogorov-Smirnov. Os valores obtidos dos testes, p $<0,05$ para as três subescalas de UDO e UDO global, indicam fuga da normalidade. Diante destes resultados, foram aplicados testes não paramétricos para verificação das hipóteses, pois estes não presumem que os dados sigam a distribuição normal. Para analisar a diferença entre dois grupos independentes foi usado o teste de Kruskal-Wallis, e para mais de dois grupos independentes, o teste de Mann-Whitney.

As variáveis sociodemográficas, após análise de frequência, foram assim categorizadas: sexo (homem, mulher), raça (brancos e não brancos), raça-sexo (homem branco, homem não branco, mulher branca e mulher não branca), escolaridade (com e sem pós-graduação), estado civil (casados, separados e solteiros), orientação sexual (heterossexual e outros), religião (católicos, protestantes, espíritas, irreligiosos e outros). As hipóteses relativas às variáveis idade e tempo como gestor (escala de razão) foram testadas pela correlação bivariável de Spearman com duas extremidades.

Todas as hipóteses foram testadas ao nível de 5\% de significância. As hipóteses 1 e 2 foram rejeitadas, significando que gestores mulheres e homens (hipótese 1), homens brancos e não brancos, mulheres brancas e não brancas (hipótese 2) não apresentam diferenças estatisticamente significativas nas atitudes em relação ao diverso (Tabela 4). Pelo resultado da estatística descritiva isso quer dizer que, de forma geral, os gestores apresentam atitudes igualmente favoráveis com relação à diversidade de contato, ao conforto com diferenças, à apreciação relativista e ao constructo UDO global.

Mesmo não sendo uma hipótese elaborada com base em evidências teóricas e empíricas, como as anteriores, devido à carência de literatura, a título de explorar os dados obtidos foram realizados testes para as variáveis escolaridade, estado civil, orientação sexual e religião. Similarmente às hipóteses anteriores, nenhuma das categorias dessas variáveis apresentou diferença estatisticamente significativa nas dimensões de UDO e UDO global. 
$\mathrm{F}$

Tabela 4 - Resultados dos testes não paramétricos

\begin{tabular}{|c|c|c|c|c|c|}
\hline $\begin{array}{l}\text { onte: } \\
\text { Elabor } \\
\text { ado }\end{array}$ & Variáveis & $\begin{array}{c}\text { Diversidade de } \\
\text { Contato }\end{array}$ & $\begin{array}{c}\text { Apreciação } \\
\text { Relativista }\end{array}$ & $\begin{array}{c}\text { Conforto } \\
\text { com } \\
\text { diferenças }\end{array}$ & $\begin{array}{l}\text { UDO } \\
\text { global }\end{array}$ \\
\hline pelos & Sexo & 0,698 & 0,110 & 0,972 & 0,370 \\
\hline autore & Raça & 0,789 & 0,211 & 0,059 & 0,918 \\
\hline s. & \$exoRaça & 0,891 & 0,170 & 0,273 & 0,655 \\
\hline ota: $\mathrm{O}$ & Escolaridade & 0,059 & 0,756 & 0,544 & 0,256 \\
\hline & Estado civil & 0,180 & 0,144 & 0,646 & 0,064 \\
\hline signifi & Orientação sexual & 0,520 & 0,787 & 0,550 & 0,548 \\
\hline 105 & Religião & 0,467 & 0,872 & 0,412 & 0,883 \\
\hline
\end{tabular}

Com relação à hipótese 3 , existe uma correlação negativa baixa, mas estatisticamente significativa, entre idade dos gestores e diversidade de contato, dimensão comportamental do constructo UDO. Isso significa que gestores mais novos apresentam maior interesse em procurar uma diversidade de experiências sociais e culturais em que haja um senso de interação com diferentes pessoas e práticas. Contrariamente, gestores com mais idade apresentam menos interesse na interação com diversos. Resultado similar foi obtido com a UDO global (Tabela 5).

A hipótese 4, experiência como gestor, apresentou uma correlação negativa e baixa, mas estatisticamente significativa, somente com a apreciação relativista, a dimensão cognitiva do constructo UDO (Tabela 5). Os resultados apontam que quanto mais experiência tem o gestor, menor parece ser a sua compreensão sobre pessoas com outras origens étnicas e raciais. Na medida em que gestores com mais experiência tendem a ter mais idade, esse resultado condiz com o obtido na hipótese 3.

Tabela 5 - Coeficientes de correlação de Spearman

\begin{tabular}{|c|c|c|c|c|c|}
\hline Variáveis & Coeficiente correlação & $\begin{array}{l}\text { Diversidade de } \\
\text { Contato }\end{array}$ & $\begin{array}{c}\text { Apreciação } \\
\text { Relativista }\end{array}$ & $\begin{array}{c}\text { Conforto } \\
\text { com } \\
\text { diferenças }\end{array}$ & $\begin{array}{l}\text { UDO } \\
\text { global }\end{array}$ \\
\hline \multirow[t]{2}{*}{ Idade } & Spearman &,$- 106^{*}$ &,- 078 &, 013 &,$- 103^{*}$ \\
\hline & Sig. (2-extremidades) & ,020 & ,088 & ,771 & ,023 \\
\hline \multirow[t]{2}{*}{ Tempo como gestor } & Spearman &,- 031 &,$- 092^{*}$ & ,079 &,- 045 \\
\hline & Sig. (2-extremidades) & ,500 & ,044 & 083 & ,325 \\
\hline
\end{tabular}

Nota:* Correlação é significativa no nível de 0.05 (2-caudas). $\mathrm{N}=484$.

Fonte: Elaborado pelos autores.

\section{DISCUSSÃO DOS RESULTADOS}

Com respeito à hipótese 1 , contrariamente às evidências empíricas e argumentações teóricas formuladas, as mulheres gestoras não apresentaram atitudes UDO mais elevadas que seus pares homens, tanto na UDO global como nas três subescalas. De forma geral, pelo fato de as mulheres serem mais favoráveis a ações voltadas à diversidade do que os homens (KLUEGEL; SMITH, 1983; MOR-BARAK, 2000) no ambiente organizacional era esperado que as gestoras tivessem atitudes UDO mais positivas que os homens. No contexto acadêmico predominantemente estadunidense, vários estudos que aplicaram a M-GUDS, completa ou 
reduzida (MIVILLE et al., 1999; STRAUSS; CONNERLEY, 2003; SAWYERR; STRAUSS; YAN, 2005; STRAUSS; SAWYERR; OKE, 2008; TOSCANO, 2012) evidenciaram correlações positivas com a UDO. O resultado da pesquisa demonstra que gestores mulheres e homens apresentam igualmente atitudes UDO, ou seja, não diferem em relação às atitudes de apreciação de similaridades e diferenças entre pessoas, no tocante aos componentes afetivo, cognitivo e comportamental do constructo UDO.

Em relação à hipótese 2, contrariamente ao pressuposto, gestores brancos, sejam mulheres ou homens, não apresentaram escores de M-GUDS-S mais elevados que gestores (homens ou mulheres) não brancos. Para indivíduos pertencentes a grupos raciais minoritários, seria esperado que apresentassem maior nível de UDO, em razão da maior probabilidade de terem contato com pessoas de fora do seu grupo racial (FUERTES et al., 2000). No entanto, tais autores não encontraram diferenças significativas entre distintos grupos raciais nas atitudes UDO global de estudantes universitários. Todavia, Strauss e Connerley (2003) e Strauss, Sawyerr e Oke (2008) identificaram que estudantes não brancos demonstravam atitudes mais favoráveis ao diverso do que estudantes brancos, em subescalas da UDO ou UDO global. Toscano (2012) encontrou uma relação negativa entre estudantes brancos e atitudes UDO. É preciso destacar que a amostra é constituída por 77,7\% de brancos e $19,2 \%$ de negros (pardos e pretos). Essa composição majoritária poderia explicar um potencial etnocentrismo do grupo majoritário (homens brancos) em relação aos demais grupos sociais. Etnocentrismo tem sido definido como "a tendência para ver membros do próprio grupo (endogrupo) como o centro do universo, para interpretar outros grupos sociais (exogrupo) e avaliar crenças, comportamentos e valores do próprio grupo, de certa forma, mais positivamente que aqueles do exogrupo" (SHIMP; SHARMA, 1987, apud COX, 1993, p.130).

A hipótese 3 foi parcialmente verificada, pois foi demonstrada uma correlação negativa e significativa da idade com diversidade de contato e UDO global. Assim, os gestores mais jovens tendem ao interesse e comprometimento em participar de atividades sociais e culturais, com foco internacional e diverso. Alguns indicadores apontam que os Millennials têm atitudes de abertura para questões de diversidade e justiça social (COOMERS; DEBARD, 2004), confirmando pesquisa sobre valores que demonstrou que pessoas mais jovens atribuíam maior importância a valores de abertura à mudança e menor ao conservadorismo do que pessoas mais velhas (SCHWARTZ, 1992). Ao mesmo tempo, a correlação com UDO global mostra que gestores mais jovens exibiram atitudes de compreensão e de aceitação, tanto de similaridades como de diferenças entre pessoas, levemente mais elevadas que gestores mais velhos. Em pesquisas com estudantes, Strauss e Connerley (2003) constataram a existência de correlação positiva entre idade e atitudes UDO na subescala conforto com diferenças, o que não foi observado nesta pesquisa.

Com relação à hipótese 4 , os gestores menos experientes tendem a apresentar atitudes mais favoráveis que os mais experientes, na dimensão afetiva da UDO. Essas atitudes refletem a apreciação de similaridades e de diferenças entre pessoas e o impacto de tais similaridades e diferenças na autocompreensão e crescimento pessoal. Este resultado condiz com a afirmação de Forteza e Prieto (1994), de que o tempo de experiência dos indivíduos tende a influenciar suas reações cognitivas e afetivas no ambiente de trabalho. 


\section{CONCLUSÕES}

As atitudes UDO dos gestores, de forma geral, foram elevadas e não discriminadas por gênero e raça, nas dimensões cognitiva, comportamental e afetiva. Miville et al. (1999) entendem que uma pessoa com elevada UDO pode buscar uma diversidade de experiências com outros (comportamental) porque valoriza similaridades e diferenças entre ela e os outros (cognitiva). Essas experiências podem reforçar os valores de UDO e resultar em um senso de conexão com outros (emocional). Nesse sentido, os gestores de nossa pesquisa são capazes de construir conexões, ser mais abertos e expressar mais sensibilidade com pessoas culturalmente diversas. Os mesmos autores evidenciaram uma relação negativa entre UDO e atitudes dogmáticas e homofóbicas. Desse resultado, poder-se-ia inferir que indivíduos com elevada UDO teriam pensamentos menos rígidos e fechados em seu sistema de valores, e menos homofóbicos. Este é o aspecto positivo. No entanto, uma análise crítica aponta para a necessidade de considerar as diferenças amostrais entre esta pesquisa e aquelas realizadas principalmente nos Estados Unidos, além das diferenças culturais entre os dois países, que poderiam ter eventuais efeitos sobre os resultados.

Os resultados deste estudo têm implicações teóricas e práticas. De uma perspectiva teórica, a escala M-GUDS-S, que mensura o constructo UDO, teve validação convergente, discriminante e confiabilidade satisfatórias para uma amostra de 484 gestores brasileiros, com restrição para um parâmetro da validade convergente, que apontou cargas fatoriais abaixo de 0,7 em alguns itens da escala. Alguns resultados de estudos realizados com amostra unicamente de estudantes universitários dos Estados Unidos e Inglaterra não foram confirmados. Uma possível razão seria o fato de a amostra deste estudo apresentar perfil diferenciado, constituída por respondentes mais velhos (73,8\% na faixa entre 30 e 49 anos), predominantemente homens $(71,9 \%)$ e brancos $(77,7 \%)$, com formação superior e pósgraduação $(86,4 \%)$. A amostra foi composta por gestores nos cargos de Supervisão, Gerência e Direção, com tempo médio como gestor de 9,3 anos, caracterizados como profissionais experientes. Além desse fato, cabe salientar que a pesquisa foi realizada em uma única empresa - corporação multinacional estadunidense de grande porte do setor de agronegócios e de alimentos, presente nas cinco regiões geográficas do Brasil. Além de um perfil homogêneo, constatou-se que as respostas também tenderam para um mesmo padrão, mais concentradas em níveis mais elevados de UDO, sem distinção entre gestores homens e mulheres, e brancos e não brancos.

O conteúdo dos itens da M-SGUDS-S é genérico quanto à dimensão da diversidade, não abordando questões específicas em relação a gênero, orientação sexual, raça e idade, uma vez que é previsto que atitudes UDO sejam relevantes para pessoas de todas as raças (MIVILLE et al., 1999, p. 293). Dois itens sobre pessoas com deficiência se referem ao aprendizado que elas podem proporcionar (Pessoas com deficiência podem me ensinar coisas que eu não poderia aprender com outras pessoas - subescala apreciação relativista) e à intenção de estar com essas pessoas (Eu me interessaria em participar de atividades que envolvessem pessoas com deficiência - subescala diversidade de contato), como seres humanos que apresentam tanto similaridades como diferenças em relação a si próprios. No Brasil foi instituída a Lei 8.213/91 (Lei de Cotas), com o objetivo de estabelecer cotas de vagas no mercado de trabalho para Pessoas com Deficiência (PcDs). Esta lei exige que a empresa contrate PcDs e acidentados, conforme o número de funcionários na organização. Nesse sentido, os gestores experientes provavelmente têm em suas equipes PcDs e aprenderam a lidar com elas.

Cinco itens da M-GUDS-S (subescalas conforto com diferenças e diversidade de contato) abordam de forma ampla questões raciais, referindo-se a: [...] raça diferente da minha; [...] pessoas da minha própria raça; [...] pessoas de outra raça; [...] pessoas de 
diferentes origens raciais, e [...] pessoas de raças diferentes. Ou seja, nenhum item trata especificamente da raça negra ou outra. Uma implicação crítica que se poderia depreender é que, para uma sociedade miscigenada como a brasileira, eventualmente a M-GUDS poderia não estar capturando apropriadamente as atitudes voltadas aos aspectos raciais, principalmente na dimensão conforto com diferenças (aspecto emocional da UDO) e diversidade de contato (aspecto comportamental da UDO). Logo, estudos futuros são necessários, com diferentes amostras, inclusive com estudantes, a fim de analisar os resultados e, eventualmente, promover ajustes na M-GUDS-S.

Uma implicação prática diz respeito à possibilidade de usar a M-GUDS-S como instrumento de diagnóstico para apoiar as políticas de diversidade, programas de treinamento, de expatriação e de carreiras, assim como para um acompanhamento longitudinal das atitudes dos gestores.

\section{REFERÊNCIAS}

BRISLIN, R. W. Back-translation for cross-cultural research. Journal of Cross-Cultural Psychology, v. 1, n. 3, p. 185-216, 1970.

CHIN, W. W.; NEWSTED, P. R. Structural equation modeling analysis with small samples using partial least squares. In: HOYLE, R. H. (Ed.). Statistical strategies for small sample research. Thousand Oaks, CA: Sage Publications, 1999, p. 307-341.

COOMERS, M. D.; DEBARD, R. (Eds.). Serving the millennial generation. New Directions for Student Services, v. 68, n. 106. Hoboken, NJ: John Wiley \& Sons, 2004.

COX, T. H. Cultural diversity in organizations: theory, research and practice. San Francisco, CA: Berrett-Koehler Publishers, 1993.

COX, T. H.; BLAKE, S. Managing cultural diversity: implications for organizational competitiveness. Academy of Management Executive, v. 5, n. 3, p. 45-56, 1991.

EUROPEAN SOCIAL SURVEY EDUCATION NET. First round of preparation, cleaning and recoding. Disponível em: <http://essedunet.nsd.uib.no/cms/topics/1/4/2.html >. Acesso em: 26 out. 2012

FISHBEIN, M.; AJZEN, I. Belief, attitude, intention and behavior: an introduction to theory and research. Reading, MA: Addison-Wesley Publishing Co., 1975.

FLEURY, M. T. L. Gerenciando a diversidade cultural: experiências de empresas brasileiras. RAE - Revista de Administração de Empresas, v.40, n. 3, p. 18-25, 2000.

FORTEZA, J. A.; PRIETO, J. M. Aging and work behavior. In: TRIANDIS, H. C.; DUNNETTE, M. D.; HOUGH, L. M. Handbook of Industrial and Organizational Psychology. 2. ed. Palo Alto, CA: Consulting Psychologists Press, 1994. v. 4. p. 447-483.

FRANÇA, C. B.; LOURENÇO, P. R. Diversity and intragroup conflict at work: an empirical study in Portugal. RAM - Revista de Administração Mackenzie, v.11, n.3, p. 130-158, 2010. 
FUERTES, J. N. et al. Factor structure and short form of the Miville-Guzman UniversalityDiversity Scale. Measurement and Evaluation in Counseling and Development, v. 33, n.3, p. 157-169, 2000.

GOUVEIA, V. V. et al. Escala de desejabilidade social de Marlowe-Crowne: Evidências de sua validade fatorial e consistência interna. Avaliação Psicológica, v. 8, n. 1, p. 87-98, 2009.

GUILLEMIN, F.; BOMBARDIER, C.; BEATON, D. Cross-cultural adaptation of healthrelated quality of life measures: Great Britain, literature review and proposed guidelines. Journal of Clinical Epidemiology, v. 46, n. 12, p. 1417-1432, 1993.

HAIR, J. F. et al. A primer on Partial Least Squares Structural Equation Modeling (PLSSEM). Los Angeles, CA: Sage, 2014.

HAIR, J. F. et al. Fundamentos de métodos de pesquisa em administração. Porto Alegre: Bookman, 2005.

HANASHIRO, D. M. M.; CARVALHO, S. G. Diversidade cultural: panorama atual e reflexões para a realidade brasileira. REAd - Revista Eletrônica de Administração, edição 47, v.11, n.5, 2005.

HARRISON, D. A.; KLEIN, K. J. What is the difference? Diversity constructs as separation, variety, or disparity in organizations. Academy of Management Review, v.32, n. 4, p.11991228, 2007.

HOMAN, A. C. et al. Bridging faultlines by valuing diversity: Diversity beliefs, information elaboration, and performance in diverse work groups. Journal of Applied Psychology, v. 92, n. 5, p. 1189-1199, 2007.

JACKSON, S.; MAY, E.; WHITNEY, K. Understanding the dynamics of diversity in decision-making teams. In: GUZZO, R.; SALAS, E. (Eds.). Team decision making effectiveness in organizations. San Francisco, CA: Jossey-Bass, 1995.

JUNG, C. G. Os arquétipos e o inconsciente coletivo. Petrópolis: Vozes, 2000.

KLUEGEL, J. R.; SMITH, E. R. Affirmative action attitudes: effects of self-interest, racial affect, and stratification beliefs on white's views. Social Forbes, v. 61, n. 3, p. 797-824, 1983.

KOHLI, H. K. et al. Universal-diverse orientation of business, education, and social work students in a north-eastern comprehensive university. International Journal of Management in Education, v.10, n.2, p. 111-130, 2016.

KOSSEK, E. E.; ZONIA, S. C. Assessing diversity climate: A field study of reactions to employer efforts to promote diversity. Journal of Organizational Behavior, v. 14, n. 1, p. 6181, 1993.

LINNEHAN, F. et al. Behavioral goals for a diverse organization: the effects of attitudes, social norms, and racial identity for Asian Americans and Whites. Journal of Applied Behavioral Psychology, v. 33, n. 7, p. 1331-1359, 2003. 
LODEN, M.; ROSENER, J. Workforce America! Managing employee diversity as a vital resource. New York: McGraw-Hill, 1991.

MANNIX, E.; NEALE, M.A. What differences make a difference? Psychological science in the public interest. v.6, n.2, p. 31-55, 2005.

MIVILLE, M. L. et al. Appreciating similarities and valuing differences: The Miville-Guzman universality diversity scale. Journal of Counseling Psychology, v. 46, n. 3, p. 291-307, 1999.

MIVILLE, M. L. et al. Universal-diverse orientation: linking social attitudes with wellness.

Journal of College Student Psychotherapy,v.19, n.2, p.61-79, 2004.

MOR-BARAK, M. E. Beyond affirmative action: toward a model of diversity and organizational inclusion. Administration in Social Work., v. 23, n. 3/4, p. 47-68, 2000.

MOR-BARAK, M. E. Managing diversity: toward a globally inclusive workplace. Thousand Oaks, CA: Sage Publications, 2005.

NKOMO, S. M.; COX, T. Diversidade e identidade nas organizações. In: CLEGG, S.R.; HARDY, C.; NORD, W. R. (Eds.). Handbook de Estudos Organizacionais. São Paulo: Atlas, 1999. v. 1.

PEDHAZUR, E. J. Multiple regression in behavioral research: explanation and prediction. 3. ed. New York: Wadsworth, 1997.

PEREIRA, J. B. C.; HANASHIRO, D. M. M. Escala de atitudes diante de ações organizacionais em prol da valorização da diversidade. Psychologica, v. 55, p. 167-187, 2011.

PEREIRA, J. B. C.; HANASHIRO, D. M. M. Ser ou não ser favorável às práticas de diversidade? Eis a questão. RAC - Revista de Administração Contemporânea, v. 14, n. 4, p. 670-683, 2010.

PODSAKOFF, P. M.; MACKENZIE, S. B.; PODSAKOFF, N. P. Common method biases in behavioral research: A critical review of the literature and recommended remedies. Journal of Applied Psychology, v. 88, n. 5, p. 879-903, 2003.

QIN, J.; MUENJOHN, N.; CHHETRI, P. A review of diversity conceptualization: variety, trends, and a framework. Human Resource Development Review. v. 13, n.2, p. 133-157, 2014.

RINGLE, C. M.; DA SILVA, D.; BIDO, D. Modelagem de equações estruturais com utilização de SMARTPLS. ReMark - Revista Brasileira de Marketing, (Ed. Especial), v. 13 , n. 2, p. 56-73, 2014

ROBERTS, J.A.; LAUX, J.M.; BURCK, A.M. Exploring the relationship between UniversalDiverse Orientation and personality. Journal of Counseling Practice, p. 1-7, 2009.

SAWYERR, O. O.; STRAUSS, J.; YAN, J. Individual value structure and diversity attitudes. The moderating effects of age, gender, race, and religiosity. Journal of Managerial Psychology, v. 20, n. 6, p. 498-521, 2005. 
SCHWARTZ, S. H. Universals in the content and structure of values: Theoretical advances and empirical tests in 20 countries. Advances in Experimental Social Psychology, v. 25, p. $1-65,1992$.

SINGLEY, D. B; SEDLACEK, W. E. Differences in universal-diverse orientation by raceethnicity and gender. Journal of Counseling and Development. v.84, n.4, p. 404-411. 2009.

STRAUSS, J. P.; CONNERLEY, M. I. Demographics, personality, contact, and universaldiverse orientation: an exploratory examination. Human Resource Management, v. 42, n. 2, p. 159-174, 2003.

STRAUSS, J. P.; SAWYERR, O. O.; OKE, A. Demographics, individual value structures, and diversity attitudes in the United Kingdom. Journal of Change Management, v. 8, n. 2, p. 147-170, 2008.

THOMAS JR., R. R. Redefining diversity. New York: AMACOM, 1996.

TOSCANO, L. A. The influence of diversity experiences on undergraduate students' universal diverse orientation (UDO). Doctor of Philosophy Degree in Higher Education. The University of Toledo, 2012.

VONTRESS, C. E. A personal retrospective on cross-cultural counseling. Journal of Multicultural Counseling \& Development, v. 24, n. 3, p. 156-166, 1996.

VONTRESS, C. E. Cross-cultural counseling: an existential approach. Journal of Counseling \& Development, v. 58, n. 2, p. 117-122, 1979.

WILLIAMS K. Y.; O'REILLY III, C.A., Demography and diversity in organizations: a review of 40 years on research. In: STAW, B.M; CUMMINGS, L.L.(Eds.). Research in Organizational Behavior. Greenwich, CT: JAI Press. v. 20, 1998. 